children and adolescent age groups. Males predominated and mental retardation and cerebral palsy were the most frequently associated conditions.

\title{
VARIABIIITY OF SEIZURE CLASSIFICATION
}

One senior neurologist (JBB) from the Department of Neurology and Pediatrics, West Virginia University, Morgantown, W.Va., USA, and three neurology residents from the University of Oklahoma, Harvard Unitersity, and $\mathrm{W} . \mathrm{Va}$. University reviewed descriptions of seizures transcribed from medical records and classified them according to the International League Against Epilepsy (ILAE) Classification system, 1981. The overall agreement in classifying seizure types by the ILAE system was relatively poor and only slightly better than would have been expected by chance. Agreement was particularly weak for atypical absence, partial seizures with secondary generalization, and generalized motor seizures. It was better for simple and complex partial, simple absence and infantile spasms. Approximately $22 \%$ of descriptions were insufficient for classification by the ILAE system. The authors conclude that the development and testing of more explicit criteria for the diagnosis of specific seizure types may be especially useful in improving the reliability of seizure classification. (Bodensteiner JB et al. Interobserver variability in the ILAE classification of seizures in childhood. Epilepsia March/April 1988; 29: 123-128).

COMMRN. A classification of seizure types is of little value without assessment of its reproducibility in practice. Surprisingly, this study appears to be the first test of the reliability of the ILAE system of seizure classification, first proposed in 1970 and generally employed in statistical trials of anticonvulsant drugs. The relatively low level of concordance among observers using the ILAE system in the present study is disturbing, and the substitution of a more specific seizure classification should be entertained especially in therapeutic trials.

\section{PSYCHOLOGICAL TREATMENT OF EPILEPSY}

Three children, ages 12-15, with frequent refractory seizures were treated by behavioral intervention techniques in the Depts of Medical Rehabilitation and Clinical Neurophysiology, Orebro Medical Center Hospital, Orebro, and Dept of Clinical Psychology, University of Uppsala, Sweden. Seizure types described as myoclonic in pt 1, Jacksonian (pt 2), and minor motor (pt 3) were associated with mental deterioration. Behavioral countermeasures, consisting of tensing of muscle groups and screaming "stop"! (pt 1), massaging the affected limb (pt 2), and fixing eye movements on an object (pt 3), resulted in an immediate reduction in both the seizures and paroxysmal EEG activity in all three cases. Other behavioral strategies, including biofeedback training in awareness of early seizure signals, applied relaxation, and positive reinforcement, were without beneficial effect. The authors propose that countermeasures in the level and speed of arousal may be the mechanism of the treatment intervention. (Dahl J, Melin L, Leissner P. Effects of behavioral intervention on epileptic seizure behavior and parosysmal activity: a systematic replication of three cases of children with intractable epilepsy. Epilepsia March/April 1988; 29: 172-183). 